\title{
Former des professionnels via la simulation : confrontation des principes pédagogiques issus de la littérature et des pratiques de terrain
}

How to train professionals through simulation-based training. A comparison between pedagogical principles from research and field practices

\section{Zoya Horcik}

\section{OpenEdition}

Journals

Édition électronique

URL : http://journals.openedition.org/activites/963

DOI : 10.4000/activites.963

ISSN : $1765-2723$

Éditeur

ARPACT - Association Recherches et Pratiques sur les ACTivités

\section{Référence électronique}

Zoya Horcik, «Former des professionnels via la simulation : confrontation des principes pédagogiques issus de la littérature et des pratiques de terrain », Activités [En ligne], 11-2 | Octobre 2014, mis en ligne le 15 octobre 2014, consulté le 30 avril 2019. URL : http://journals.openedition.org/activites/963

DOI : 10.4000/activites.963

\section{c.) (i) (9)}

Activités est mis à disposition selon les termes de la licence Creative Commons Attribution - Pas d'Utilisation Commerciale - Pas de Modification 4.0 International. 


\title{
Former des professionnels via la simulation : confrontation des principes pédagogiques issus de la littérature et des pratiques de terrain
}

\author{
Zoya Horcik \\ Faculté de psychologie et de sciences de l'éducation, 40 boulevard Du Pont d'Arve, 1211 Genève 4 (Suisse) \\ Zoya.horcik@unige.ch
}

\begin{abstract}
How to train professionals through simulation-based training. A comparison between pedagogical principles from research and field practices. Simulation-based training is becoming a dominant model in healthcare education. The aim of this paper is to discuss the traditional theoretical frameworks used in simulation-based training and the activity analysis principles which guided our research. This paper is based on two types of intervention that were conducted in the simulation centre at Geneva University hospital (SimulHUG): the first related to research conducted among anaesthetist nurses engaged in a vocational training program using fullscale simulation sessions. The second related to a working group which involved trainers who used simulation basedtraining, and researchers. These working groups were initiated to draw links between the debriefing, as currently practised in the institution, and the principles of activity analysis. We believe that the issues raised by the trainers with whom we have worked can enlighten and nourish discussion on the use of activity analysis for vocational training. We present four main issues raised by the trainers: a) confidentiality; b) how to capture the activity of the trainees, c) the use of video and d) how to speak about marginal or non-prescribed practices that can appear during the debriefing.
\end{abstract}

KEYWORDS

simulation, activity analysis, debriefing, trainers, experience

\section{Introduction}

De nombreuses institutions hospitalières se sont dotées au cours de ces dernières années de simulateurs et de centres de simulation pour former leur personnel. Bien que la simulation soit utilisée depuis de nombreuses années dans le domaine des soins, les progrès techniques, la standardisation des cursus de formation au niveau national et le développement exponentiel de la littérature au niveau international à ce sujet ont conduit à une généralisation des pratiques de formation par simulation entre les différents sites hospitaliers. Ces dispositifs de formation réorganisent de façon importante les cursus et méthodes traditionnelles de formation et nécessitent que les formateurs soient eux-mêmes formés à la conception et l'utilisation de ces nouveaux outils.

Initialement développé pour les domaines à risques (Grau, Doireau, \& Poisson, 1998), un des objectifs des formations par simulation est le maintien des performances des professionnels dans des situations routinières ou dégradées comme dans le cas de 
catastrophes mettant en jeu plusieurs corps de métiers (Aggarwal, Undre, Moorthy, Vincent \& Darzi, 2005). Dans le milieu médical, le développement de simulateurs partiels de hautefidélité permet aux médecins de travailler sur la reconnaissance de certaines pathologies reproduites par le simulateur et sur des manipulations techniques complexes, parfois impossibles à effectuer dans des conditions de travail réel (Van der Meijden \& Schijven, 2009) pour des raisons éthiques ou sécuritaires (Ziv, Root, Wolpe, Small, \& Glick, 2003). Les patients standardisés (acteur simulant un certain nombre de symptômes) sont particulièrement utilisés dans le domaine de la médecine de premier recours, médecine générale, ou la psychiatrie. Les simulateurs de pleine échelle reproduisent à la fois les environnements professionnels de manière exhaustive et la dynamique des situations ce qui a pour effet l'apparition de la thématique de la communication au sein des équipes (Gaba, Howard, Fish, Smith, \& Sowb, 2001).

La littérature décrivant les formations par simulation (Fanning \& Gaba, 2007) identifie trois phases dans l'organisation de ces sessions : la première concerne la phase «du briefing " durant laquelle les formateurs accueillent les participants, rappellent les règles d'usage du simulateur et l'organisation de la session de simulation. La deuxième phase est celle de la «simulation» proprement dite au cours de laquelle les participants jouent ce que nous nommons un scénario simulé préparé à l'avance par les formateurs. Enfin la troisième partie concerne le «debriefing » au cours duquel les participants reviennent sur le scénario qui a été simulé. Le debriefing peut être appuyé ou non par un support vidéo.

Cette dernière phase est considérée comme la phase la plus importante de la session de formation dans la mesure où elle concentrerait l'apprentissage en simulation. La littérature sur les formations par simulation fournit des supports aux formateurs pour la conception et l'animation des debriefings (Chronister \& Brown, 2012 ; Decker, Fey, Sideras, Caballero, Rockstraw, Boese, Franklin, et al., 2013). Ils font l'objet d'un nombre important de recherches et de comptes rendus qui en détaillent le déroulement, les types de relances ou questionnements à adresser aux participants (Fanning \& Gaba, 2007; Rudolph, Simon, Dufresne, \& Reamer, 2006). Toutefois, malgré l'abondance de littérature à ce sujet, les recherches et collaborations que nous avons pu mener au sein du centre de simulation (SimulHUG) des Hôpitaux Universitaires des Genève, montrent des problématiques spécifiques que nous pensons résulter d'une tension entre les conceptions de l'apprentissage des adultes qui sous-tendent les formations par simulation et la nature de l'activité simulée telle que nous l'avons mise en avant dans nos recherches qui concernaient l'analyse de l'activité réelle des participants à ce type de dispositif (Horcik \& Durand, 2011).

Nous avons mené deux types d'intervention au sein du centre de simulation des Hôpitaux Universitaires de Genève (SimulHUG) et sur lesquelles se basent cet article : la première était liée à une recherche effectuée auprès d'infirmiers anesthésistes engagés dans un cursus de formation qui incluait des sessions sur un simulateur de pleine-échelle (c'est-à-dire, un simulateur reproduisant l'environnement physique et la dynamique situationnelle en salle d'opération). Cette recherche avait pour objectif de documenter l'activité réelle des infirmiers en simulation. Elle nous a permis de développer un certain nombre d'hypothèses qui questionnent la conception générale de formations par simulation et qui sont relayées cidessous. La seconde intervention était liée au développement de groupes de travail composés de formateurs chargés d'animer des formations par simulation et de chercheurs. Ces groupes de travail ont été initiés afin de développer les liens entre la pratique du debriefing, tel qu'il est pratiqué actuellement dans l'institution, et l'analyse de l'activité.

L'objectif de cet article est de mettre en discussion les cadres théoriques traditionnels utilisés au cours des formations par simulation et les principes d'analyse de l'activité qui guident nos recherches. Nous pensons que la mise en perspective des problématiques soulevées par les formateurs avec qui nous avons collaboré peut éclairer et nourrir la discussion sur l'usage des techniques de l'analyse de l'activité pour la formation professionnelle. 


\section{1.- Le debriefing : un élément fondamental des formations par simulation}

Nous nous basons sur une revue non exhaustive de la littérature centrée sur le debriefing pour résumer les principales caractéristiques et les cadres théoriques en guident la conception. La littérature sur ce sujet peut être synthétisée en deux parties : la première concerne des études évaluant l'efficacité des debriefings en fonction de différents paramètres comme la structure du debriefing ou l'utilisation de supports audio-vidéos (Chronister \& Brown, 2012 ; Welke, Leblanc, Savoldelli, Joo, Chandra, Crabtree, \& Naïk, 2009), la seconde est constituée de revues de littérature ou de comptes rendus élaborant une série de «bonnes pratiques » à adopter pour conduire un debriefing (Fanning \& Gaba, 2007 ; Gardner, 2013; Neil \& Wotton, 2011; Welke et al., 2009). Ce type de recherche, majoritairement à destination des formateurs et concepteurs de formation par simulation, est fondé sur des principes dits «evidence based « (c'est-à-dire d'études effectuées de manière systématique en double aveugle et mettant en jeu des groupes expérimentaux et des groupes de contrôles, pré-tests et post-tests) qui guident la recherche en médecine (Decker et al., 2013) et la conception des cursus de formation via des collaborations internationales comme le courant BEME (Best Evidence Medical Education). Ce courant recense, via des revues de littérature, les recherches expérimentales apportant des preuves de l'efficacité de méthodes, concepts pour l'éducation dans le milieu médical (http://www.bemecollaboration.org/Home/).

\section{1.- Résumé de la littérature sur le debriefing}

Le debriefing tel qu'il est décrit dans la littérature, trouve son origine dans le domaine militaire puis dans le domaine des soins où il est d'abord utilisé après des incidents critiques ou des évènements spécifiques à des fins stratégiques et thérapeutiques (Fanning \& Gaba, 2007). Avec le développement des formations par simulation, sa pratique devient systématique et il est aujourd'hui considéré comme constituant la phase la plus importante des formations par simulation (Gardner, 2013 ; Mayville, 2011 ; Welke et al. 2009). Des études montrent également que les scénarios simulés qui sont suivis d'un debriefing donnent de meilleurs résultats après les formations que ceux qui n'ont pas suivi de debriefing (Savoldelli, Naik, Park, Joo, Chow, \& Hamstra, 2006).

La conception des debriefings s'appuie notamment sur les travaux de Kolb (1984) et l'apprentissage expérientiel (Argyris \& Schön, 1996 ; Zigmont, Kappus, \& Sudikoff, 2011). De manière générale, cette théorie se base sur le fait que l'apprentissage procède toujours à partir d'une expérimentation concrète de la part de l'acteur suivie d'un processus réflexif qui vient alimenter la construction d'abstractions généralisantes (qui correspondent à la construction de concepts). Ces abstractions sont ensuite explorées par l'acteur au cours de nouvelles expérimentations concrètes, etc. Sans debriefing, les sessions de formation par simulation se limiteraient alors à un simple exercice pratique (les scénarios simulés), où les possibilités d'apprentissage seraient réduites ou difficilement contrôlables par les formateurs. Les apports de Dewey (1938) et Knowles (1980) guident la manière dont est conduit le debriefing par les formateurs. L'apprentissage adulte y est vu comme essentiellement guidé par l'expérience, la motivation et la perception d'un gain immédiat de compétences applicables au terrain. Dans cette perspective, l'objectif de la session de formation par simulation est de faire prendre conscience aux participants des raisons qui ont guidé leurs choix.

Pastré (2005) insiste également sur le caractère essentiel du debriefing pour l'apprentissage en simulation, très utilisé dans les recherches en didactique professionnelle. Pour trois raisons principales : premièrement l'activité simulée met l'accent sur le pôle productif de l'activité et ceci particulièrement quand le scénario présente des problèmes typiques, et ne laisse que peu de temps au participant pour émettre un pronostic sur la situation en cours. Le 
debriefing permet alors de jouer sur l'aspect temporel et mettre l'accent sur le pôle constructif de l'activité. Deuxièmement, le debriefing opère également comme reconstruction de l'activité comme une «mise en intrigue» (Ricoeur, 1986) : les participants visionnent ou se remémorent leur action à la lumière de l'action complète qu'ils ont effectuée et en connaissant par avance son dénouement. Ceci a alors pour effet de mettre en relation les actions effectuées et leur résultat à plus ou moins long terme et de favoriser ainsi la compréhension de l'action. La troisième raison concerne la capacité de généralisation des situations singulières, qui est liée à la mise en intrigue. En recherchant des enchaînements de causes à effets, le participant construit alors un champ conceptuel qui lui permettra dans un second temps d'identifier des classes de situations et de développer une reconnaissance des problèmes spécifiques.

\section{2.- Structure du debriefing}

Gardner (2013), à la suite de Fanning et Gaba (2007), décrit le debriefing comme comportant trois phases majeures. La première concerne «les réactions à chaud» qui permettent aux participants qui viennent de jouer un scénario simulé de s'exprimer sur la situation qu'ils viennent de vivre. Le formateur (ou «facilitateur» pour employer le terme qui le désigne durant le temps de la formation par simulation) cherche à identifier durant cette phase l'état émotionnel des participants. Les articles sur ce sujet mentionnent régulièrement que cette phase doit avoir lieu le plutôt possible voire immédiatement après le scénario simulé.

La deuxième phase correspond à ce que Gardner (2013) nomme la phase de « compréhension». Durant cette phase le formateur/facilitateur cherche à faire expliciter aux participants les évènements les uns après les autres et à retracer la genèse des choix qui ont été effectués au moyen de relances spécifiques et en établissant un climat de confiance (Cantrell, 2008 ; Rudolph et al., 2006 ; Wickers, 2010). Cette phase peut être appuyée par l'usage des enregistrements audio-vidéos du scénario simulé. C'est au cours de cette phase que le formateur/facilitateur intervient pour explorer les autres possibilités qui auraient pu être réalisées par les participants au cours du scénario simulé. C'est également durant cette phase qu'un apport ou un rappel plus formel des procédures est effectué si nécessaire (par exemple dans le cas d'un groupe de novices).

Enfin, la troisième phase résume la discussion qui a eu lieu durant la phase précédente. Le formateur/facilitateur demande généralement aux participants de mentionner un élément positif s'étant déroulé durant la simulation et un élément à améliorer.

\section{3.- Le formateur/facilitateur}

La fonction de facilitateur est utilisée dans la littérature afin de distinguer l'activité du formateur engagé dans l'animation des debriefings de celle du formateur « enseignant » ayant un rôle transmissif.

\footnotetext{
"Unlike the traditi' nal classr “ $m$ " teacher," facilitat $r$ s tend $t$ ` $p$ ” siti” $n$ themselves $n$ ' $t$ as auth rities ` $r$ experts, but rather as c'learners. This $m$ `re fraternal appr`ach may be $m$ `st $p r$ 'ductive where the learning 'bjective is behavi` ral change. Facilitat' $r$ s aim $t$ ' guide and direct rather than $t^{\prime}$ lecture. The $r{ }^{\prime} l e{ }^{\prime} f$ the student ' $r$ participant in debriefing is expanded fr' $m$ the traditi 'nal passive r'le $t$ ' 'ne where the skills demanded ' $f$ them are the ability $t$ ' critically analyze `ne's ‘w perf rmance retr` spectively-n` $t$ just what went well but what went wr'ng, and why it went that way, and t`c c'ntribute actively $t^{`}$ the learning pr`cess » (Fanning \& Gaba, 2007, p. 118).
}

L'alternance entre ces deux rôles peut être un objet de tension dans l'activité du formateur/facilitateur. Cette tension est clarifiée par une classification des «degrés de facilitation ». Ces degrés varient en fonction : du niveau des participants, de la nature du scénario simulé, du temps disponible, de la familiarité des participants avec la simulation, etc. Trois niveaux ont été identifiés: un niveau de facilitation élevé correspond à un debriefing mené principalement par les participants. Le facilitateur guide le debriefing, 
donne la parole, gère le temps, etc. Il s'agit d'un niveau de facilitation principalement utilisé avec des groupes «experts », de niveau professionnel avancé ou habitués aux sessions par simulation. Les relances utilisées par le formateur/facilitateur peuvent être des silences, ou des questions de clarification. Le niveau de facilitation intermédiaire spécifie que les relances se doivent d'être davantage des reformulations, des modifications de questions ou des commentaires de l'action d'un participant par un de ses collègues. Un niveau de facilitation bas indique des interventions fréquentes de la part du formateur/facilitateur. Ce niveau est requis lorsque le groupe est inexpérimenté, ou lorsqu'il répond de manière superficielle aux questions. Les relances sont des affirmations/confirmations, ou une analyse systématique de l'action. Fanning et Gaba (2007) mentionnent qu'un haut niveau de facilitation est peu fréquent et que les formateurs/facilitateurs conduisent généralement des debriefings avec un niveau de facilitation bas.

Rudolph, Simon, Dufresne et Raemer (2006) spécifient de manière plus précise la nature des relances du facilitateur qui se doivent de contraster avec la notion «d'instruction» [qui correspond à la partie transmissive du corpus de la formation, comme les procédures en vigueur (normes d'hygiène) ou les notions théoriques à connaître (calcul des doses de médicaments)]. L'idée d'environnement protégé pour les participants et la notion de «jugement positif» structure les relances: le formateur/facilitateur se doit d'éviter les questions «non jugeantes » afin d'éviter la tendance à ne pas mentionner les problèmes issus de l'activité des participants. Les relances types doivent alterner constats sur l'activité réalisée et une question. Dans ce modèle de debriefing, le formateur/facilitateur conserve son rôle d'expert, mais profite également du point de vue des participants sur le sens qu'ils donnent à leur pratique.

\section{2.- Les problématiques posées aux formateurs/facilitateurs}

Nous présentons les principales problématiques des formateurs/facilitateurs que nous avons pu relever au cours de nos différentes collaborations au sein des Hôpitaux Universitaires de Genève. L'élaboration de ces différentes problématiques est basée sur des notes et comptesrendus de séances de travail autour de la question du debriefing pour les formations par simulation.

Les formateurs/facilitateurs qui travaillaient dans le centre de simulation animaient fréquemment les sessions de formation en binôme : un formateur prenait le rôle de pilote du scénario et de formateur/facilitateur principal. Il analysait l'action générale et récoltait des éléments pour le debriefing. Le second était en général présent dans la salle où se déroulait le scénario simulé (appelée la "salle d'opération ») et endossait le rôle d'un aide-soignant, d'un chirurgien ou celui d'un autre membre du corps médical. Il pouvait donner des informations aux participants au cours du scénario (il annonce par exemple des signes que le mannequin ne peut simuler, comme la couleur de sa peau). Il repérait également des éléments pour le debriefing depuis la salle d'opération. Au cours du debriefing cette personne pouvait assurer le rôle de formateur/facilitateur secondaire (intervention sur un thème particulier ou gestion des tours de parole, du temps de la vidéo, etc.).

Nous avons identifié quatre problématiques récurrentes chez les formateurs/facilitateurs : a) la confidentialité, b) capter l'activité, c) l'usage de la vidéo et d) parler des pratiques non prescrites.

\section{1.- La confidentialité}

Le premier problème identifié par les formateurs/facilitateurs concerne celui de la confidentialité. Au début de la formation, les participants sont informés du fait que «ce qui se passe dans le simulateur reste dans le simulateur». Cette règle, essentielle au bon fonctionnement de la situation, contraint les formateurs/facilitateurs à ne pas considérer les 
évènements qui se déroulent dans le simulateur comme une évaluation sommative ou formative. C'est-à dire que les formateurs/facilitateurs ne tiennent pas compte des erreurs parfois problématiques qui sont effectuées par les participants quitte à minimiser ce qui s'est passé au cours de la simulation. Cela signifie, d'une part, que la résolution d'éventuels problèmes doit être effectuée uniquement durant le debriefing et, d'autre part, que des éléments vus en durant la simulation ne peuvent pas être réévoqués plus tard dans les cursus des participants. Ceci remet en question pour quelques formateurs/facilitateurs l'utilité des sessions de formation par simulation : comment exploiter l'activité simulée dans un cursus de formation initiale et continue soumise à cette règle de confidentialité ?

\section{2.- Capter l'activité}

Le deuxième problème est lié à la difficulté éprouvée par les formateurs/facilitateurs à capter la spécificité de l'activité des participants durant son déroulement (voir ce qu'ils font et comment ils le font). Ils perçoivent fréquemment des différences entre leur propre activité et celle des participants, mais ne parviennent pas à les identifier de manière spécifique. Ceci est en partie dû à l'enchaînement rapide des différentes phases de la session de formation. Cet enchaînement rapide est lié à deux éléments : d'une part une même session de formation (qui dure en général trois à quatre heures) prévoit souvent plusieurs scénarios et d'autre part cet enchaînement rapide est préconisé dans la littérature afin d'éviter que les participants ne commentent leurs impressions avant le debriefing. Les deux formateurs/facilitateurs doivent à la fois piloter un scénario et récolter des éléments pour l'animation du debriefing. Durant cette session, ils s'attachent également à respecter le principe selon lequel les debriefings doivent être conduits le plus rapidement possible après le scénario. Ceci laisse donc très peu de temps aux formateurs/facilitateurs pour se coordonner, et repérer les éléments spécifiques qui ont eu lieu durant le scénario.

\section{3.- L'usage de la vidéo}

Le centre de simulation étant doté de matériel d'enregistrement vidéo et de projection, l'usage de la vidéo au cours des debriefings est très fréquent. Ce recours à la vidéo incite donc les formateurs/facilitateurs à faire réagir les participants sur la base de ces enregistrements tout en essayant de maintenir des apports théoriques. L'utilisation de la vidéo fait apparaître l'activité simulée des participants qui montre les hésitations et parfois des erreurs de procédure commises par des professionnels expérimentés. Cette mise en visibilité de l'activité parfois marginale ou non prescrite ne peut être passée sous silence par les formateurs/facilitateurs, car les professionnels repèrent eux-mêmes les erreurs qu'ils ont commises et celles de leurs collègues. Les formateurs/facilitateurs sont quant à eux particulièrement sensibles à l'effet parfois « direct » que peut avoir la vidéo sur la perception que les participants ont de leur propre activité.

\section{4.- Parler des pratiques marginales ou non prescrites}

La mise en visibilité de l'activité des participants conduit à aborder des problématiques du travail qui entrent parfois en contradiction avec les procédures établies. Dans le cas des professionnels expérimentés (qui viennent au centre de simulation dans le cadre de leur formation continue) ceci peut ouvrir sur des discussions riches dans la mesure où ils connaissent et pratiquent ces procédures depuis plusieurs années, mais plus complexe en ce qui concerne des novices. Cette émergence de l'activité réelle au cours du scénario simulé ne permet pas la conception d'une progression par les formateurs/facilitateurs à destination des novices et complexifie l'animation du debriefing. Dans le cadre du cursus de formation des novices, les sessions de formation par simulation interviennent à un moment où l'enjeu principal pour les novices est de s'approprier les procédures et les «bonnes pratiques » de la profession. 
Ceci est particulièrement problématique pour les formateurs/facilitateurs (qui souvent suivent ou côtoient les participants sur le terrain) et qui incarnent le rôle de garant de ces bonnes pratiques pour les participants.

\section{3.- Mise en perspective}

Cette partie cherche à mettre en discussion les problématiques soulevées par les formateurs/facilitateurs avec le cadre théorique qui guide nos recherches. Nous faisons l'hypothèse que l'analyse de l'activité réelle peut permettre une meilleure compréhension et un développement des dispositifs de formation par simulation tels qu'ils sont actuellement dispensés dans les centres de formation médicaux.

Nos recherches s'inscrivent dans le courant général de l'analyse de l'activité issue de l'ergonomie de langue française et plus particulièrement dans le cadre théorique et méthodologique du cours d'action développé par Theureau (2006). Nos recherches cherchent à concevoir et améliorer des dispositifs de formation sur la base de l'analyse de l'activité réelle des professionnels. Ceci nous conduit à aborder les formations par simulation avec une conception de l'activité humaine fondée deux présupposés principaux: premièrement l'activité de l'acteur résulte d'un couplage autonome et asymétrique acteur-environnement, et deuxièmement elle relève d'un niveau particulier de conscience dite conscience préréflexive qui correspond au niveau élémentaire faisant expérience pour l'acteur et susceptible d'être exprimée par lui (décrit, mimé, raconté ou commenté), moyennant un certain nombre de conditions telles que, par exemple, une remise en situation dynamique à partir de traces de son activité (Horcik, Savoldelli, Poizat, \& Durand, 2014).

\section{1.- Le debriefing de type réflexif et l'activité du formateur/facilitateur}

La pratique du debriefing dans le milieu des soins est fortement ancrée dans les théories de l'apprentissage réflexif (Knowles, 1980). Un des principes fondamentaux de cette théorie réside dans l'idée que le seul fait de revoir ou réévoquer son action, expliciter les fondements de sa pensée permet le développement et l'apprentissage chez les participants. Pour les formations par simulation cela signifie que la confrontation à la vidéo et le commentaire qui en est fait pas les participants suffisent à développer leur activité. Ceci a à voir avec un problème plus large qui est celui de l'objectif du debriefing comme étant soit une instance de formation «transmissive» (le formateur est un «instructeur») soit comme une session «réflexive » (le formateur est un «facilitateur »).

Cette prédominance de l'aspect réflexif de l'apprentissage, qui induit ce type particulier d'animation, peut poser problème notamment dans les domaines à risque qui sont très fortement prescriptifs et où le rôle de la formation est de rappeler les procédures ou de transmettre les mises à jour de ces procédures. C'est pourquoi les formations par simulation ont un statut hybride qui est ressenti comme particulièrement sensible pour les formateurs/facilitateurs qui se situent entre recherche et explicitation et transmission d'expertise. Ce type de formation illustre les difficultés également rencontrées dans d'autres domaines professionnels comme le domaine scolaire. Fanning et Gaba (2007) identifient cette tension, mais la mettent directement en lien avec le degré d'expertise du formateur/facilitateur. La fonction de facilitateur est donc fortement liée à la dimension réflexive de la conception du debriefing.

\section{2.- L'activité simulée et l'activité réelle}

Nous pensons que les cadres théoriques actuels qui sous-tendent les formations par simulation opèrent une double réduction de l'activité des participants : premièrement les formations par simulation conçues selon cette orientation ne prennent pas en compte la spécificité de l'activité simulée au cours du scénario, mais constituent ce que Gardner (2013) 
a identifié comme « une excuse pour faire un debriefing 》 (p. 166). Le scénario simulé est alors principalement un pourvoyeur d'ancrages servant au debriefing. Deuxièmement les éléments observables de l'activité sont décomposés en compétences techniques, compétences communicationnelles, émotions, etc. ce qui limite selon nous la compréhension fine de cette activité.

Il nous semble que les points soulevés par les formateurs/facilitateurs concernant les difficultés relatives à l'utilisation de la vidéo et à l'émergence de pratiques non prescrites durant le debriefing peuvent toutes deux être expliquées par la nature de l'activité simulée qui est différente de l'activité de travail réelle et qui est fréquemment confondue avec celuici lors de la conception et de l'animation des sessions de formation.

Nos recherches ont porté sur l'analyse des cours d'expérience d'infirmiers-anesthésistes engagés dans des sessions de formations sur simulateurs de pleine-échelle (Horcik et al., 2014). Les résultats montrent que l'expérience des participants durant les scénarios simulés est composée en permanence d'éléments relatifs au travail réel et à l'activité en simulation et ne peut donc être réduite strictement à l'expérience de travail ou à l'expérience de formation. La structure de préparation des infirmiers entremêle en permanence des éléments liés à la situation se déroulant «ici et maintenant» (la simulation et ses composantes, l'enregistrement de la situation en cours, les interventions des formateurs/facilitateurs, la salle d'opération est une salle reconstituée, l'activité d'anesthésie effectuée sur le mannequin) et l'activité professionnelle réelle et incarnée à laquelle se réfère cette simulation. Cette expérience particulière a été qualifiée dans cette étude «d'expérience mimétique » et illustre la notion de «double intentionnalité » mise en évidence notamment dans les travaux d'anthropologie (Horcik et al., 2014 ; Willerslev, 2004).

Notre hypothèse est que les formations par simulation actuelles telles qu'elles sont conçues ne prennent pas en compte l'activité simulée en ce qu'elle est une «activité simulée réelle » c'est-à-dire une «reconstruction des conditions écologiques des situations de travail » selon Theureau (2006). Les debriefings sont menés de façon à n'évoquer presque exclusivement que le travail réel qui correspond à l'activité ciblée par la formation. La part de l'activité simulée qui ne se réfère pas directement à l'activité ciblée n'est que très rarement prise en compte et est considérée comme un élément annexe ou anodin de l'expérience. Nous pensons que cette expérience, entremêlant les deux composantes (à la fois le travail et la formation) constitue la spécificité des formations par simulation et pourrait expliquer en partie leur efficacité.

Nous pensons que la non-prise en compte de la spécificité de l'expérience simulée contribue à appauvrir le debriefing dans la mesure où il ne s'agit plus tout à fait d'apprentissage expérientiel vu que cette non-prise en compte crée une rupture dans la boucle de l'apprentissage expérientiel en orientant le debriefing non vers l'expérience vécue, mais vers l'activité que la formation vise. Dans cette optique nous avons pu observer que les scénarios simulés deviennent moins intéressants pour les formateurs/facilitateurs en raison de leur conception et organisation contraignante et du caractère imprévisible de l'activité des participants. Les scénarios simulés sont progressivement remplacés par de la vidéoformation où les formateurs/facilitateurs effectuent un scénario à l'avance et le font commenter par les participants au cours d'un debriefing. L'émergence de pratiques erronées ou transgressives pourrait également être liée à des difficultés des participants à produire une activité dans le cadre du scénario simulé et ne serait donc pas forcément due à leur incompétence ou à de la malveillance. En résumé, considérer l'activité simulée de manière globale permettrait de prendre en compte "l'activité réelle simulée » à l'instar de la distinction classique entre travail prescrit et travail réel qui fonde le courant de l'analyse de l'activité.

\section{3.- Capter l'activité des participants : la question de la temporalité}

La difficulté soulevée par les formateurs/facilitateurs concernant la gestion du temps 
restreinte entre le scénario simulé et le debriefing peut être mise en relation avec la forme des entretiens d'autoconfrontation que nous avons menés au cours de notre recherche.

La constitution de notre corpus de recherche comprenait à la fois une collecte de vidéos des scénarios simulés et d'entretiens d'autoconfrontation individuels des participants. Bien que les entretiens d'autoconfrontation restent majoritairement utilisés à des fins de recherche et qu'ils ne reposent pas sur les mêmes présupposés théoriques, on peut noter des similarités dans la forme des entretiens d'autoconfrontations et des debriefings tels qu'ils sont menés au cours des formations par simulation. Tout d'abord, l'usage de la vidéo a été systématique pour les participants que nous avons observés à la fois pour les entretiens d'autoconforntation et pour les debriefings. Les consignes et relances données par les chercheurs et le formateur/facilitateur étaient également similaires pour une partie des debriefings (le participant avait pour consigne dans les deux cas de décrire son activité et documenter les éléments ayant fait signe pour lui au moment du scénario simulé). Dans le cadre de notre recherche, les entretiens d'autoconfrontation avaient été effectués entre une semaine et un mois après les scénarios simulés pour des raisons de disponibilité des participants. Nous avons observé que le processus de remise en situation n'a pas été rendu plus difficile par le temps qui s'était écoulé depuis le jour de la formation. Ceci pourrait signifier que les debriefings pourraient être effectués sur une période plus ou moins longue après la session de simulation et autoriserait alors aux formateurs/facilitateurs un temps d'analyse plus important.

Nous avons également constaté que les participants avaient tendance à réagir non seulement à propos de l'activité que nous leur avons montrée, mais aussi à propos leur activité actuelle et les transformations de cette activité lorsque nous les interrogions plusieurs semaines après la session de formation. Par exemple, une infirmière avait immédiatement remarqué qu'une alarme s'était déclenchée sur l'enregistrement vidéo du scénario simulé, mais a indiqué que, à l'époque du scénario simulé, elle n'avait pas entendu cette alarme. Elle a mentionné avoir développé une sensibilité aux alarmes depuis l'enregistrement de ce film. Nous pensons que la réexploitation des scénarios simulés antérieurs pourrait également constituer une démarche intéressante afin de renforcer les effets des séances de formation par simulation basées sur la vidéo.

\section{4.- Éthique et image de soi}

L'utilisation régulière de la vidéo pour les debriefings introduit des questions qui nous semblent relatives à la vidéoscopie et à son usage pour la formation et la recherche (Leblanc \& Veyrunes, 2011 ; Linard \& Prax, 1985). Si dans la conception des debriefings actuels la vidéo est utilisée principalement comme trace de l'activité et support au commentaire des participants, nous pensons que cet usage dans le cadre des formations professionnelles fait accéder le professionnel à une image particulière de lui-même à laquelle il n'a quasiment jamais été confronté lors de sa pratique professionnelle (l'usage de la vidéo en formation professionnelle restant encore relativement rare). Comme le mentionnent Linard et Prax (1985), la vidéo met en scène le participant en le dépossédant de son image :

«[...] M n pr`pre c`rps n’est plus p`ur la caméra qu’un 'bjet parmi d'autres, se déplaçant dans un espace d`nt $m$ ' $n$ oeil n'est plus l’ rganisateur central et d`nt il perd le c`ntrôle» (p.175).

L'usage de la vidéo se révèle être à la fois un outil puissant et délicat à gérer dans la mesure où il instaure un jeu de proximité-distance entre le professionnel et son image au cours d'une activité qui ne correspond pas complètement à son activité habituelle puisqu'il s'agit d'une activité simulée.

L'aspect collectif du debriefing peut également poser problème dans la mesure où des jeux sociaux peuvent s'installer entre les acteurs (Theureau, 2006). Ces jeux sociaux, selon nos observations sur le terrain, surviennent en lien avec le rôle parfois peu clair des 
formateurs/facilitateurs, avec la systématicité des debriefings vidéos et également lorsque le groupe de participants comprend des professionnels venant de différents secteurs (médecins infirmiers, aides-soignants, etc.).

Les règles de confidentialité et jugement positif se doivent donc de cadrer de manière forte les interactions entre les participants. Toutefois, la complexité des phénomènes liés à l'utilisation de la vidéo et aux problématiques liées au groupe rendent les questions d'éthique, de jugement positif et de confidentialité certes nécessaires, mais non suffisantes pour une exploitation complète des vidéos dans un cadre de formation.

\section{4.- Conclusion}

Nous pensons que les simulations telles qu'elles sont exploitées actuellement constituent un outil de formation peut-être plus complexe qu'il n'y paraît au regard de la littérature sur ce sujet. L'activité simulée, prise pour l'activité réelle (à la fois pour les formateurs/facilitateurs et les participants) peut conduire à des difficultés d'analyse de cette même activité ou à des situations qui exposent les participants à des problèmes d'image parfois difficiles à gérer.

À mi-chemin entre la recherche et la formation, l'analyse et l'exploitation de l'activité en formation se situent au cœur des pratiques des formateurs-chercheurs et les liens qui les relient nécessitent d'être explorés de manière plus approfondie.

\section{BIBLIOGRAPHIE}

Aggarwal, R., Undre, S., Moorthy, K., Vincent, C., \& Darzi, A. (2005). The simulated operating theatre: Comprehensive training for surgical teams. Quality and Safety in Health Care, 13(1), 2732 .

Argyris, C., \& Schön, D. (1996). Organizati nal Learning: The`ry, Meth`d, and Practice. Reading MA: AddisonWesley.

Cantrell, M.A. (2008). The importance of debriefing in clinical simulation. Clinical Simulati $n$ in Nursing, 4(2), 19-23.

Chronister, C., \& Brown, D. (2012). Comparison of simulation debriefing methods. Clinical Simulati $n$ in Nursing, 8(7), 281-288.

Decker, S., Fey, M., Sideras, S., Caballero, S., Rockstraw, L., Boese, T., Franklin, A., Gloe, D., Lioce, L, Sando, C., Meakim, C., \& Borum, J. (2013). Standards of best practice: simulation standard VI: The debriefing process. Clinical Simulati $n$ in Nursing, 9(6) 26-29.

Dewey, J. (1938). Experience and Educati` $n$. NewYork: Macmillan.

Fanning, R.M. \& Gaba, D.M. (2007). The role of debriefing in simulation based learning. Simulati $n$ in Healthcare, 2(2), 115-125.

Gaba, D.M., Howard, S.K., Fish, K.J., Smith, B.E., \& Sowb, Y.A. (2001). Simulation-based training in anaesthesia crisis resource management (ACRM): A decade of experience. Simulati $n \&$ Gaming, 32(2), 175-193.

Gardner, R. (2013). Introduction to debriefing. Seminars in Perinat l' gy, 37, 166-174.

Grau, J.Y., Doireau, P., \& Poisson, R. (1998). Conception et usage de la simulation. Le Travail Humain, 61(4), 361-385.

Horcik, Z., \& Durand, M. (2011). Une démarche d'ergonomie de formation un projet pilote en formation par simulation d'infirmiers anesthésistes. Activités, 8(2), 173-188. http://www .activites.org/v8n2/v8n2.pdf

Horcik, Z., Savoldelli, G.L., Poizat, G., \& Durand, M. (2014). A phenomenological approach to novice nurse anesthetists' experience during simulation-based training sessions. Simulati $n$ in Healthcare, 9(2), 94-101.

Knowles, M.-S. (1980). The M`dern Practice `f Adult Educati`n: Fr`m Pedag 'gy t`Andrag`gy. San Francisco CA: Jossey-Bass.

Kolb, D.A. (1984). Experiential Learning: Experience as the s`urce`flearning and devel pment. Englewood Cliffs,N J: Prentice-Hall. 
Leblanc, S, \& Veyrunes, P. (2011). «Vidéoscopie » et modélisation de l'activité enseignante. Recherche et frmati $n, 68,139-152$.

Linard, M., \& Prax, I. (1985). Images vide` images de s`i... `u Narcisse au travail. Paris: Dunod.

Mayville, M.-L. (2011). Debriefing, the essential step in simulation. Newb`rn and Infant Nursing Reviews, 11(1) 35-39.

Neil, A., \& Wotton, K. (2011). High-fidelity simulation debriefing in nursing education: A literature review. Clinical Simulati $n$ in Nursing, 7(5) 161-168.

Pastré, P. (2005). Apprendre par la résolution de problèmes : le rôle de la simulation. In P. Pastré \& P. Rabardel (Eds.), Appendre par la simulati $n$ : De l'analyse du travail aux apprentissages pr`fessi` nnels, (pp. 17-40). Toulouse: Octarès.

Ricoeur, P. (1986). Du texte à l'acti` $n$. Paris: Seuil.

Rudolph, J.W., Simon, R., Dufresne, R.L., \& Reamer, D.B. (2006). There's no such thing as a“"nonjudgmental"debriefing: A theory and method for debriefing with good judgment. Simulati $n$ in Healthcare, 1, 49-55.

Savoldelli, G., Naik, V.N., Park J., Joo, H.S., Chow, R., \& Hamstra, S.J. (2006). Value of debriefing during simulated crisis management: Oral versus video-assisted oral feedback. Anesthesi $l$ ' $g y$, 105(2), 279-285.

Theureau, J. (2006). Le c`urs d'acti n. Méth`de dével ppée. Toulouse: Octarès.

Van der Meijden, O.A.J., \& Schijven, M.P. (2009). The value of haptic feedback in conventional and robot-assisted minimal invasive surgery and virtual reality training: A current review. Surgical End sc`py, 23(6), 1180-1190.

Welke, T., Leblanc, V.R., Savoldelli, G.L., Joo, H-S., Chandra, D.-B., Crabtree, N.A., \& Naïk, V.N. (2009). Personalized oral debriefing versus standardized multimedia instruction after patient crisis simulation. Ec` $n^{`}$ mics, Educati n, and P`licy, 109(01), 183-189.

Wickers, P. (2010). Establishing the climate for a successful debriefing. Clinical Simulati $n$ in Nursing, 6(3), 83-86.

Willerslev, R. (2004). Not animal, not not-animal: hunting imitation and empathetic knowledge among the Siberian Yukaghirs. J urnal `f the R`yal Anthr` $p$ ' ${ }^{\prime}$ gical Institute, 10(3), 629-652.

Zigmont, J., Kappus, L., \& Sudikoff, S. (2011). Theoretical foundations of learning through simulation. Seminars in Perinat' $l$ ' gy, 35, 47-51.

Ziv, A., Root Wolpe, P., Small, S.D., \& Glick, S. (2003). Simulation-based medical education: An ethical imperative. Academic Medicine 78(8), 783-788.

\section{RESUME}

Les formations par simulation sont en train de devenir un modèle de formation dominant dans le domaine de la santé. L'objectif de cet article est de mettre en discussion les cadres théoriques traditionnels utilisés au cours des formations par simulation et les principes d'analyse de l'activité qui guident nos recherches. Nous avons mené deux types d'intervention au sein du centre de simulation des Hôpitaux Universitaires de Genève (SimulHUG) et sur lesquelles se base cet article: la première était liée à une recherche effectuée auprès d'infirmiers anesthésistes engagés dans un cursus de formation sur un simulateur de pleine échelle. La seconde était liée à un groupe de travail composé de formateurs chargés d'animer des formations par simulation et de chercheurs. Ces groupes de travail ont été initiés afin de développer les liens entre la pratique du debriefing, tel qu'il est pratiqué actuellement dans l'institution, et les principes de l'analyse de l'activité. Nous pensons que la mise en perspective des problématiques soulevées par les formateurs avec qui nous avons collaboré peut éclairer et nourrir la discussion sur l'usage des techniques de l'analyse de l'activité pour la formation professionnelle. Nous présentons les principales problématiques des formateurs/facilitateurs que nous avons pu relever. Nous en identifions quatre : a) la confidentialité ; b) capter l'activité ; c) l'usage de la vidéo et d) parler des pratiques marginales ou non prescrites. 


\section{MOTS CLES}

simulation, analyse de l'activité, debriefing, formateurs, expérience

\section{REFERENCEMENT}

Horcik, Z. (2014). Former des professionnels via la simulation : confrontation des principes pédagogiques issus de la littérature et des pratiques de terrain. Activités, 11(2), 76-86. http://www .activites.org/v11n2/v11n2.pdf

Article soumis le 17 janvier 2014, accepté pour publication le 20 mars 2014 